\title{
Regenerator Placement with Guaranteed Connectivity in Optical Networks
}

\author{
Marcio S. Savasini ${ }^{1}$, Paolo Monti ${ }^{2}$, Marco Tacca ${ }^{2}$, \\ Andrea Fumagalli ${ }^{2}$, and Helio Waldman ${ }^{1}$ \\ 1 Optical Networking Lab \\ Faculdade de Engenharia Eletrica e de Computacao \\ State University of Campinas, Campinas, SP, Brazil \\ \{savasini, waldman\}@decom.fee.unicamp.br \\ 2 Open Networking Advanced Research (OpNeAR) Lab \\ Erik Jonsson School of Engineering and Computer Science \\ The University of Texas at Dallas, Richardson, TX, USA \\ \{paolo, mtacca, andreaf\}@utdallas.edu
}

\begin{abstract}
The problem of minimizing the number of optical nodes with signal regeneration capability can be constrained to guarantee a desired degree of end-to-end connectivity in the all-optical transport network. The problem can be formulated using a $k$-connected, $k$-dominating node set, which is a known approach in mobile ad hoc wireless networks. This paper presents a preliminary study aimed at establishing whether efficient centralized solutions to this problem in optical networking ought to be investigated to improve the decentralized solutions already available for wireless networks.
\end{abstract}

Index Terms- Regenerator Placement, Connected Dominating Set, k-Connectivity, Greedy Algorithm, Optical Networks.

\section{Introduction}

Optical networks can provide transport capabilities to routers (and other electronic nodes) via the combination of optical circuits, or lightpaths [1], and optical signal regenerators. The former reduce the required number of total transponders in the network, while the latter regenerate the optical signal when it is weakened by transmission impairments [2]. If well designed, this approach may lead to a substantial cost reduction of the transport network $[3,4]$.

The transmission impairments of the optical medium limit the number of network elements that can be traversed by the optical signal without making use of regenerators [2], i.e., the maximum reach of the lightpath. In practice, one can define for each (optical) node the transparency island (TI) [5,6] as the subset of nodes that can be reached all-optically — at a given minimum transmission rate — without a significant degradation of the signal quality, e.g., bit error rate. If the two end nodes that must be connected are not within each other's 
TI, the optical signal must be regenerated at some intermediate node(s). One type of regeneration is reamplification, reshaping and retiming (3R) [7]. This type of regeneration may take place in both the electronic (OEO conversion) or the optical domain [8]. Either solution adds to the overall network cost.

Assume that both the optical topology and nodes are given. Then a cost function to be minimized is the number of regenerators, which are required in the given optical topology to provide end-to-end connectivity. Some solutions addressing this problem can be found in the literature. For example, minimal cost placement algorithms [9] compute which subset of nodes must be provided with regeneration capability to guarantee connectivity against any single link failure in the network. Blocking probability (when attempting to create end-toend connections) due to the lack of available regenerators can be minimized by special algorithms for placing regenerators [10-12]. The regenerator placement problem has some similarities with the wavelength converter placement problem and solutions devised to deal with the latter problem may be used to handle the former $[13,14]$. None of these solutions, however, looks into the regenerator placement problem while guarantying an arbitrary degree of end-to-end connectivity in the optical network.

The preliminary study in this paper tackles the problem of minimizing the number of (optical) nodes that must be enabled with 3R capability, while guarantying a desired minimum degree of end-to-end (optical) connectivity. More formally, the $3 \mathrm{R}$ nodes must be placed to obtain a $k$-connected, $k$-dominating 3R-node set (k-CD3S), while minimizing the number of 3R-nodes in the set. With a guaranteed end-to-end $k$ connectivity, the resulting transport network offers both known resilience benefits against 3R-node failures and known load balancing benefits when routing and setting up connections.

The problem of minimizing the size of k-CD3S is found to be the same problem already defined in mobile ad hoc wireless networks [15], whereby a subset of nodes is selected to form a routing backbone with a guaranteed degree of end-to-end connectivity. In the wireless network the $k$-connected, $k$-dominating set is found using a decentralized algorithm to cope with the mobility of the nodes. The k-CD3S minimization problem in optical networks, on the contrary, is solved in this paper by using a centralized algorithm that is expected to be more efficient, when compared to the decentralized one. A lower bound on the size of k-CD3S is provided, to assess the efficiency of both algorithms.

As discussed in the paper, it appears that centralized solutions to address the problem of minimizing k-CD3S are worth exploring as they yield results that are substantially closer to the lower bound, when compared to the decentralized solutions devised to solve the same problem in mobile ad hoc wireless networks.

\section{Network Model and Problem Definition}

This section contains the assumptions and properties of the (optical) networks under investigation, along with the problem definition. 
A network with arbitrary physical topology is considered. Every node in the network functions as OXC. Only a subset of the nodes has regeneration capabilities, i.e., the 3R-nodes.

The physical topology is modeled as a graph $G(\mathcal{N}, \mathcal{A})$, where $\mathcal{N}$ is the set of nodes in the network and $\mathcal{A}$ is the set of directed (fiber) links connecting the nodes. Each node is uniquely identified, e.g., node $i$ is denoted as $N_{i}$. The link connecting node $N_{i}$ to $N_{v}$ is denoted as $l_{(i, v)}$. Let $C_{(i, j)}^{(r)}$ be the set of all links and nodes that can be used to establish a lightpath ${ }^{3}$ connecting node $N_{i}$ to $N_{j}$ with transmission rate $r$, without requiring $3 \mathrm{R}$. Set $C_{(i, j)}^{(r)}$ defines a subgraph of $G(\mathcal{N}, \mathcal{A})$. Let $T I_{i}^{(r)}$ be the transparency island of node $N_{i}$, for lightpaths operating at transmission rate $r . T I_{i}^{(r)}$ is a subgraph of $G$, defined as

$$
T I_{i}^{(r)}=\cup_{j \in \mathcal{N}}\left(C_{(i, j)}^{(r)}\right) .
$$

In summary, lightpaths originating at $N_{i}$, operating at transmission rate $r$, and not requiring $3 \mathrm{R}$ can only be simple paths in $T I_{i}^{(r)}$. Note that incoming links $l_{(j, i)}$ are not in $T I_{i}^{(r)} \forall r, j$, as lightpaths containing loops are not allowed.

A second graph, i.e., $G^{\prime(r)}\left(\mathcal{N}, \mathcal{A}^{\prime(r)}\right)$, is used to represent the nodes' connectivity based on their own TI's. Let set $\mathcal{A}^{\prime(r)}$ be

$$
\mathcal{A}^{\prime(r)}=\bigcup_{i \in \mathcal{N}} \bar{l}_{(i, j)}^{(r)}, \forall j \in T I_{i}^{(r)} .
$$

In simple terms, if $\bar{l}_{(i, j)}^{(r)} \in \mathcal{A}^{\prime(r)}$ it is possible to set up a lightpath from node $N_{i}$ to $N_{j}$ without requiring $3 \mathrm{R}$. Notice that $\mathcal{A}^{\prime(r)}$ is a function of the employed transmission rate $r$. To simplify the notation in the remainder of the paper, it is assumed that all lightpaths operate at the same rate $r$, and index $r$ is dropped, e.g., $G^{\prime(r)}\left(\mathcal{N}, \mathcal{A}^{\prime(r)}\right)$ is simply denoted as $G^{\prime}\left(\mathcal{N}, \mathcal{A}^{\prime}\right)$.

1-connectivity from node $N_{i}$ to $N_{j}$ in $G^{\prime}\left(\mathcal{N}, \mathcal{A}^{\prime}\right)$ is defined as follows. Let $\mathcal{S}_{3 R} \subseteq \mathcal{N}$ be the set of 3R-nodes. $N_{i}$ is 1-connected to $N_{j}$ if there exists a simple path [16] $p=<N_{i}, N_{a_{1}}, N_{a_{2}}, \ldots, N_{a_{m-1}}, N_{j}>$ in $G^{\prime}\left(\mathcal{N}, \mathcal{A}^{\prime}\right)$, such that $\left(N_{a_{1}}, N_{a_{2}}, \ldots, N_{a_{m-1}}\right) \in \mathcal{S}_{3 R}$. Note that intra TI $(m=1)$ connectivity does not require $3 \mathrm{R}$-nodes. Inter TI connectivity requires that the lightpath signal be regenerated at selected 3R-nodes, which form a backbone for the optical signal to propagate and reach the end node. $G^{\prime}\left(\mathcal{N}, \mathcal{A}^{\prime}\right)$ is 1-connected if all the pairs $\left(N_{i}, N_{j}\right), i \neq j \in \mathcal{N}$ are 1-connected.

$N_{i}$ is $k$-connected to $N_{j}$ if there exist $k$ node-disjoint simple paths $p^{(v)}=<$ $N_{i}, N_{a_{1}^{(v)}}, N_{a_{2}^{(v)}}, \ldots, N_{a_{m}^{(v)}}, N_{j}>, v=1,2, \ldots, k$ in $G^{\prime}\left(\mathcal{N}, \mathcal{A}^{\prime}\right)$, such that $\left(N_{a_{1}^{(v)}}, N_{a_{2}^{(v)}}, \ldots, N_{a_{m}^{(v)}{ }_{m}{ }^{m}}\right) \in \mathcal{S}_{3 R}, \forall v=1,2, \ldots, k . G^{\prime}\left(\mathcal{N}, \mathcal{A}^{\prime}\right)$ is $k$-connected if all node pairs $\left(N_{i}, N_{j}\right), i \neq j \in \mathcal{N}$ are $k$-connected.

Set $\mathcal{S}_{3 R}$ is a $k$-connected, $k$-dominating $3 \mathrm{R}$-node set (k-CD3S) of $G^{\prime}\left(\mathcal{N}, \mathcal{A}^{\prime}\right)$, if and only if the following constraints are met:

\footnotetext{
${ }^{3}$ It is assumed that if a lightpath can be established, then its performance in terms of bit error rate is satisfactory.
} 
(a) $k$-dominating constraint: each node that is not in the $3 \mathrm{R}$-node set must be intra TI connected to at least $k$ R-nodes, i.e.,

$$
\sum_{j \in \mathcal{S}_{3 R}} \mathcal{L}_{(i, j)} \geq k, \forall i \in\left(\mathcal{N} \backslash \mathcal{S}_{3 R}\right)
$$

where $\mathcal{L}_{(i, j)}$ is a binary variable defined as:

$$
\mathcal{L}_{(i, j)}= \begin{cases}1 & \text { if } \bar{l}_{(i, j)} \in \mathcal{A}^{\prime} \\ 0 & \text { otherwise }\end{cases}
$$

(b) $k$-node connectivity constraint: subgraph $\tilde{G}\left(\mathcal{S}_{3 R}, \tilde{\mathcal{A}}\right)$, where

$$
\tilde{\mathcal{A}}=\left\{\bar{l}_{i, j} \in \mathcal{A}^{\prime}: i, j \in \mathcal{S}_{3 R}\right\}
$$

must be $k$-node connected. A graph is defined to be $k$-node connected if and only if the removal of any of its $k-1$ nodes does not cause a partition [16].

A centralized algorithm able to find a 3R-node placement such that $\mathcal{S}_{3 R}$ satisfies both constraints $(a)$ and $(b)$, while minimizing the number of 3R-nodes, is presented next.

\section{A Centralized Algorithm to Compute k-CD3S}

In this section a two-step approach, called Select-\&-Prune (S-\&-P), is presented to find a sub-optimal solution to the $\mathrm{k}$-CD3S problem with minimum number of 3R-nodes. In step 1, an initial solution for the $\mathrm{k}$-CD3S problem is found, by selecting a number of nodes that are potential candidates for the $3 \mathrm{R}$-node set. In step 2, a greedy algorithm is applied to prune from the initial set of 3Rnodes as many nodes as possible without violating the $k$-connected, $k$-dominating constraints on k-CD3S. Table 1 contains a pseudo code description of the two sequential steps.

\subsection{Step 1: Select}

The objective of this step is to find an initial set of 3R-nodes that may be included in k-CD3S of $G^{\prime}\left(\mathcal{N}, \mathcal{A}^{\prime}\right)$. This initial selection consists of two substeps. In the first substep, a set of $3 R$-nodes $\left(\mathcal{S}_{3 R}\right)$ if found solving a variation of the vertex cover problem. In the second substep, the just computed $\mathcal{S}_{3 R}$ is checked to verify the $k$-node connectivity constraint defined in Section 2. If $\mathcal{S}_{3 R}$ is $k$-connected, no further action is required in step 1. Conversely, if $\mathcal{S}_{3 R}$ is not $k$-connected additional nodes are sequentially selected and added to $\mathcal{S}_{3 R}$, until the $k$-node connectivity constraint is met. The two substeps are described next. 
Substep 1.1: Vertex Covering : the objective of this step is to find a set $\left(\mathcal{S}_{3 R} \subseteq \mathcal{N}\right)$, with minimum number of $3 \mathrm{R}$-nodes, that is a solution for the following problem:

$$
\min \sum_{i \in \mathcal{N}} r_{i}
$$

subject to:

$$
\sum_{j, \mathcal{L}_{(i, j)} \neq 0} r_{j} \geq k, \quad \forall i \in \mathcal{N} ;
$$

where $r_{i}$ is a binary variable defined as:

$$
r_{i}= \begin{cases}1 & \text { if } N_{i} \in \mathcal{S}_{3 R} \\ 0 & \text { otherwise }\end{cases}
$$

Note that constraint (7) is more stringent than the $k$-dominating constraint in (3). Constraint (7) requires all 3R-nodes $\in \mathcal{S}_{3 R}$ to have at least $k$ 3R-node neighbors themselves. Since the objective of the S-\&-P algorithm is to find kCD3S, this additional requirement may increase the probability that the resulting $\mathcal{S}_{3 R}$ is $k$-connected.

The size of $\mathcal{S}_{3 R}$ computed so far represents a lower bound (LB) on the size of k-CD3S. This claim is based on the following simple observation. $\mathcal{S}_{3 R}$ is by definition an optimal solution under constraint (7), i.e., (a) defined in Section 2. Then, the optimal solution under both constraints $(a)$ and $(b)$ defined in Section 2 must require the same number of $3 \mathrm{R}$-nodes or more.

Substep 1.2: Connectivity Check : the objective of this step is to check the connectivity degree of $\mathcal{S}_{3 R}$ and, if necessary, to select additional 3R-nodes for $\mathcal{S}_{3 R}$ so that both constraints $(a)$ and $(b)$ defined in Section 2 are satisfied.

This step, first checks if set $\mathcal{S}_{3 R}$ generated by the vertex covering set is $k$ connected. If $\mathcal{S}_{3 R}$ is $k$-connected the algorithm stops and set $\mathcal{S}_{3 R}$ is returned as the optimal solution of the minimum k-CD3S problem.

If set $\mathcal{S}_{3 R}$ is not $k$-connected, a node is selected from $\left(\mathcal{N} \backslash \mathcal{S}_{3 R}\right)$ and added to $\left.\mathcal{S}_{3 R}\right)$ as follows. For each source destination pair in $G^{\prime}\left(\mathcal{N}, \mathcal{A}^{\prime}\right)$ a $k$ node-disjoint shortest path [16], with weights $0\left(\forall N_{i} \in \mathcal{S}_{3 R}\right)$, weight $2 \cdot|\mathcal{N}|\left(\forall N_{i} \in\left(\mathcal{N} \backslash \mathcal{S}_{3 R}\right)\right)$ and weight $1\left(\forall l_{(i, j)} \in \mathcal{A}^{\prime}\right)$, is computed. This choice of weights forces the shortest path algorithm to compute paths that make use of already selected 3R-nodes as much as possible. All nodes $N_{i} \in\left(\mathcal{N} \backslash \mathcal{S}_{3 R}\right)$ are then scored based on the number of times they are chosen for a shortest path. The node with the highest score - i.e., the node most used in connecting source destination pairs — is added to $\mathcal{S}_{3 R}$. This process is repeated until $\mathcal{S}_{3 R}$ is $k$-connected.

\subsection{Step 2: Prune}

In this final step, the algorithm attempts to remove as many nodes from $\mathcal{S}_{3 R}$ as possible without violating both constraints $(a)$ and $(b)$. Nodes in $\mathcal{S}_{3 R}$ are first 
sorted randomly. In that order, an attempt to prune each node from $\mathcal{S}_{3 R}$ is made as follows. If removing the node from $\mathcal{S}_{3 R}$ does not violate both constraints $(a)$ and $(b)$ defined in Section 2, the node is removed permanently. Otherwise, the node is labeled permanently to remain in $\mathcal{S}_{3 R}$. Once the pruning is complete, $\mathrm{k}-\mathrm{CD} 3 \mathrm{~S}=\mathcal{S}_{3 R}$.

Table 1. Pseudo code of the Select-\&-Prune algorithm

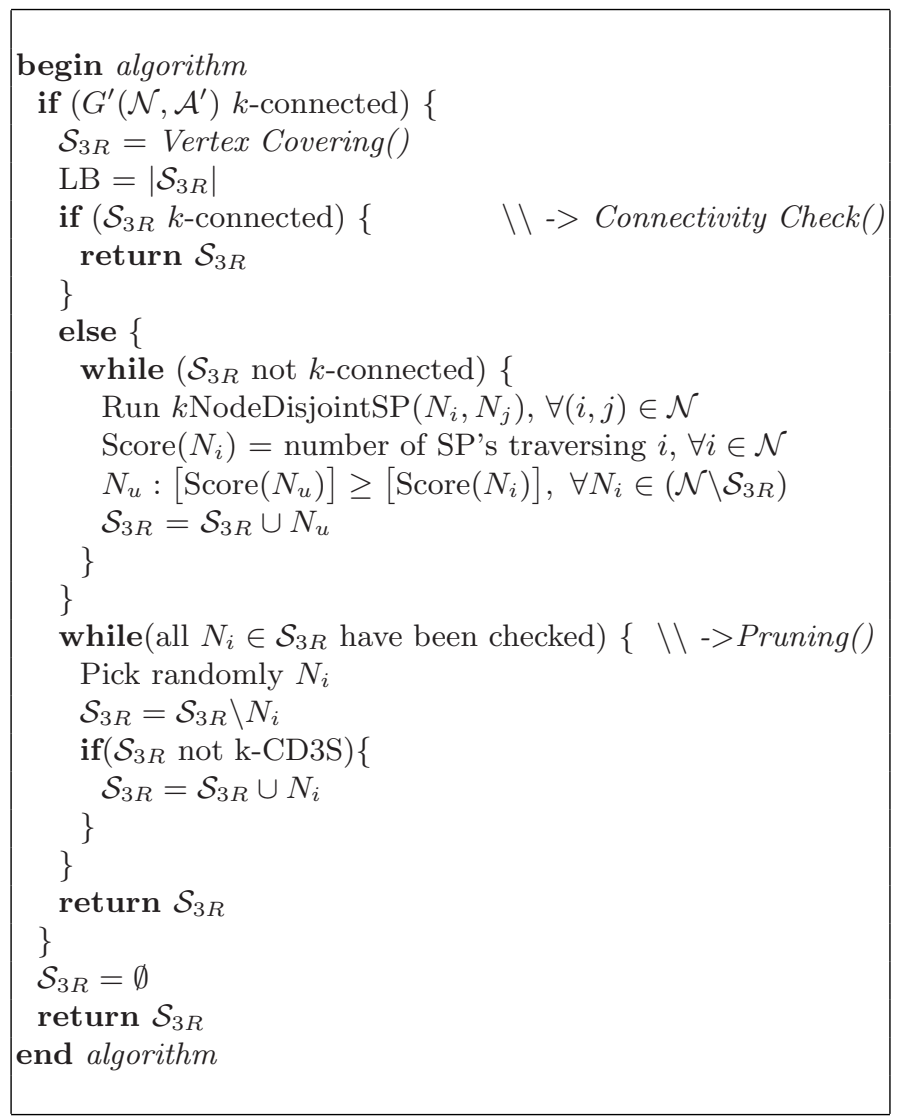

\section{Simulation Results}

This section presents a collection of simulation results obtained for the Select\&-Prune algorithm presented in Section 3. The results are compared against both the lower bound (LB) given in Section 3.1, and the solution found using the decentralized algorithm $k$-coverage proposed for mobile and ad hoc wireless networks [15]. Results for the k-coverage algorithm are obtained by first selecting 
$\mathcal{S}_{3 R}=\mathcal{N}$, and then pruning $\mathcal{S}_{3 R}$ as described in Section 3.2. The comparison is carried out to assess whether there is a substantial advantage in devising centralized solutions for the problem at hand. The ILP formulation, presented in Section 3.1 is solved using LP Solve 5.5.0.10 [18].

A number of simulation experiments is performed using network topologies that have the same minimum and average nodal degree (2, 3 respectively) and varying number of nodes. Each network topology is randomly generated using the Doer and Leslie's formula [19]. The total number of experiments is chosen so that the presented average values have a confidence interval of $12 \%$ or better at $90 \%$ confidence level. The TI of each node is chosen based on the following simplistic assumption: any lightpath without regeneration can span at most $T I=1,2,3$, and 4 physical links. The value of $T I$ is varied to analyze its effect on the algorithms' efficiency. The algorithms' performance is assessed in terms of the total number of 3R-nodes that are necessary to obtain a $k$-connected and $k$ dominating 3R-backbone. This value is normalized to the total number of nodes, i.e., the $3 R$-percentage is defined as $\% 3 R=\left|\mathcal{S}_{3 R}\right| /|\mathcal{N}|$.

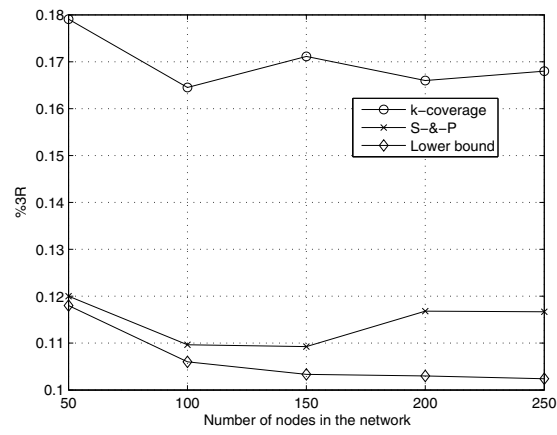

(a) $k=2$

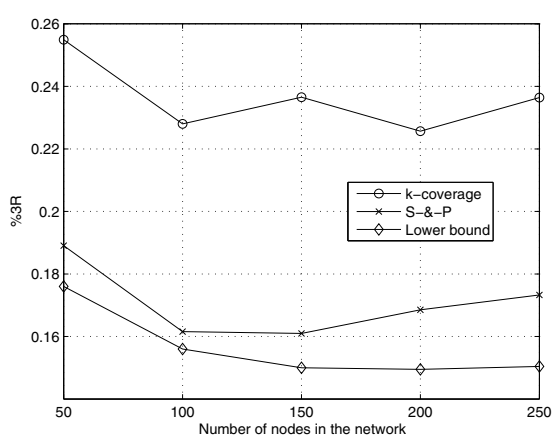

(b) $k=3$

Fig. 1. Percentage of 3R-nodes (\%3R) vs. the network size $(|\mathcal{N}|), T I=3$.

Fig. 1 shows the value of $\% 3 R$ as a function of the number of nodes in the network, when $T I=3$. Two degrees of connectivity are considered: $k=2$ (Fig.1(a)) and $k=3$ (Fig. 1(b)). In both of the cases, the centralized S-\&-P algorithm yields better results, when compared to the decentralized k-coverage algorithm. In Fig. 1(a) the difference between the lower bound and the S-\&$\mathrm{P}$ algorithm is around 1\%, while the k-coverage algorithm percentage averages almost $7 \%$ higher. Similar results are found when the required degree of connectivity increases to $k=3$ (Fig. 1(b)). In general, the two figures confirm that the number of $3 \mathrm{R}$ nodes selected by the decentralized k-coverage algorithm is at least $40 \%$ higher when compared to the centralized S-\&-P algorithm. This result suggests that a careful selection of the initial solution for k-CD3S may 
lead to a substantial cost reduction in terms of number of $3 \mathrm{R}$-nodes required in the 3R-backbone.

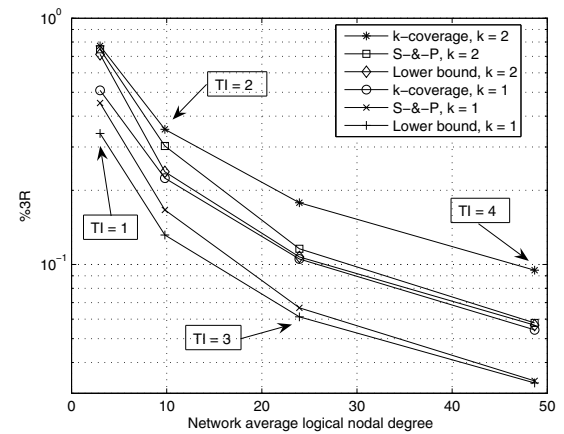

(a) $k=1$ and $k=2$

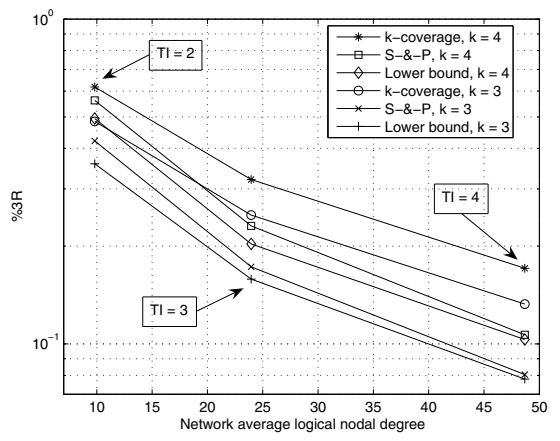

(b) $k=3$ and $k=4$

Fig. 2. Total number of $3 R$-nodes $(\% 3 R)$ vs. the average nodal degree in $G^{\prime}$.

Fig. 2 shows the value of $\% 3 R$ as a function of the average logical nodal degree in $G^{\prime}$. The nodal degree is a function of the TI size, which is reported on the plots. Four degrees of connectivity are presented: $k=1$ and $k=2$ in Fig. 2(a), $k=3$ and $k=4$ in Fig. 2(b). Note that when $T I=1, G^{\prime}=G$. Then the average nodal degree for the two graphs is 3 , making it impossible ${ }^{4}$ to provide a guaranteed degree of connectivity $k=4$ in $G^{\prime}$. As intuition suggests, the higher the value of the nodal degree the smaller is the number of required 3R-nodes. The centralized algorithm is consistently more efficient than the decentralized one. The figures also show that with approximately the same number of 3R-nodes the centralized algorithm guarantees a connectivity that is one degree higher than the one provided by the decentralized algorithm.

\section{Conclusion}

Based on the preliminary study presented in this paper, it appears that centralized algorithms may offer a substantial efficiency improvement (up to $40 \%$ in topologies with up to 250 nodes) over the already existing decentralized algorithms, when it comes to finding a sub-optimal solution to the problem of minimizing the $k$-connected, $k$-dominating node set. This result suggests that it may be worth investigating centralized algorithms in more details, as their improved efficiency translates directly into a reduction of the number of nodes with signal regeneration capability that are required in the all-optical transport network to guarantee a desired degree of end-to-end connectivity.

${ }^{4}$ All generated networks had at least one node with nodal degree $=2$, making it impossible to provide also a guaranteed degree of connectivity $k=3$. 
Acknowledgments This research was supported in part by NSF Grant No. CNS-043593, CAPES Process No. BEX4403/05-3, and the Italian Ministry of University (MIUR) (contract \# RBNEO1KNFP).

\section{References}

1. I. Chlamtac, A. Ganz, and G. Karmi, "Lightpath communications: an approach to high-bandwidth optical WAN's," IEEE Trans. Commun. 40, pp. 1171-1182, July 1992.

2. B. Ramamurthy, H. Feng, D. Datta, J. Heritage, and B. Mukerjee, "Transparent vs. opaque vs. translucent wavelength-routed optical networks," in Optical Fiber Communication (OFC'99) Technical Digest, San Diego, CA, Feb. 1999,

3. R. Ramaswami, "Optical networking technologies: What worked and what didn't," IEEE Commun. Mag. 44, pp. 132-139, September 2006.

4. I. Cerutti and A. Fumagalli, "Traffic grooming in static wavelength division multiplexing networks," IEEE Commun. Mag. 43, pp. 101-107, January 2005.

5. A. A. M. Saleh, "Islands of transparency - an emerging reality in multiwave optical networking," in 11th Annual Meeting IEEE Lasers and Electro-Optics Society, 1998.

6. J. Strand and A. Chiu, "Impairments and other constraints on optical layer routing," RFC 4054, Internet Engineering Task Force, 2005.

7. R. Ramaswami and K. N. Sivarajan, Optical Networks: a Practical Perspective, Morgan Kaufmann Publishers, 2002.

8. H.-P. Nolting, "All-optical 3R-regeneration for photonic networks," in ONDM 2003: Proceedings of the 7th IFIP Working Conference on Optical Network Design Er Modelling, 2003.

9. E. Yetginer and E. Karasan, "Regenerator placement and traffic engineering with restoration in GMPLS networks," Photonic Network Communications 2, pp. 139 149, September 2003.

10. S.-W. Kim, S.-W. Seo, and S. C. Kim, "Regenerator placement algorithms for connection establishment in all-optical networks," in IEEE Global Telecommunications Conference, 2, pp. 1205-1209, Nov. 2000.

11. X. Yang and B. Ramamurthy, "Sparse regeneration in a translucent WDM optical network," in Proceedings of APOC 2001, pp. 61-70, 2001.

12. G. Shen, W. D. Grover, T. Hiang Cheng, and S. K. Bose, "Sparse placement of electronic switching nodes for low blocking in translucent optical networks," Journal of Optical Networking, vol. 1, Issue 12, p.424 1, Dec. 2002.

13. S. Subramaniam, M. Azizoglu, and A. Somani, "On optimal converter placement in wavelength-routed networks," INFOCOM 1, pp. 500-507, Apr. 1997.

14. X. Chu, B. Li, and I. Chlamtac, "Wavelength converter placement under different RWA algorithms in wavelength-routed all-optical networks," IEEE Trans. Commun. 51, pp. 607-617, Apr. 2003.

15. F. Dai and J. Wu, "On constructing $k$-connected $k$-dominating set in wireless ad hoc and sensor networks," Journal of Parallel and Distributed Computing 66, pp. 947-958, July 2006.

16. T. H. Cormen, C. E. Leiserson, R. L. Rivest, and C. Stein, Introduction to Algorithms (Second Edition), MIT Press, Cambridge, MA, 2001.

17. M. R. Garey and D. S. Johnson, Computers and Intractability: A Guide to the Theory of NP-Completeness, W. H. Freeman \& Co., New York, NY, USA, 1979. 
18. "Lp solve 5.5.5.10." http://lpsolve.sourceforge.net/5.5/.

19. M. Doar and I. M. Leslie, "How bad is naive multicast routing?," in INFOCOM, 1, pp. 82-89, March/April 1993. 